\title{
Profile of venetoclax and its potential in the context of treatment of relapsed or refractory chronic lymphocytic leukemia
}

This article was published in the following Dove Press journal:

OncoTargets and Therapy

7 February 2017

Number of times this article has been viewed

\author{
Henriette Huber \\ Simone Edenhofer \\ Sven Estenfelder \\ Stephan Stilgenbauer \\ Department of Internal Medicine III, \\ Ulm University, Ulm, Germany
}

Correspondence: Stephan Stilgenbauer Department of Internal Medicine III, Ulm University, 23 Albert-Einstein-Allee, Ulm 8908I, Germany

$\mathrm{Tel}+4973150045521$

Fax +49 73। 50045525

Email stephan.stilgenbauer@

uniklinik-ulm.de

\begin{abstract}
Over the last few years, dramatic changes have occurred in the treatment of chronic lymphocytic leukemia (CLL). The current standard for young and fit patients with CLL remains chemoimmunotherapy, namely the fludarabine, cyclophosphamide, and rituximab (FCR) regimen. However, novel oral therapies are presently being introduced and represent a considerable breakthrough concerning effectiveness and safety profile. In particular, the very high-risk group of CLL patients, defined by the genetic aberration del(17p) and/or TP53 mutation, benefit from the new agents. These genetic abnormalities are the most relevant negative prognostic markers in the context of chemoimmunotherapy. New targeted therapies allow different approaches to improve outcomes.
\end{abstract}

Keywords: CLL, del(17p), TP53, BCL-2, venetoclax

\section{Introduction}

Chemoimmunotherapy (especially the combination of the CD20 antibody rituximab with fludarabine-cyclophosphamide [FCR]) is the standard therapy in fit (low score on the Cumulative Illness Rating Scale, normal creatinine clearance) and previously untreated patients without adverse genetic markers, such as del(17p) and/or TP53 mutation, in the frontline setting. ${ }^{1-4}$ The deletion of the short arm of chromosome 17 is defined as "del(17p)": it affects the tumor-suppressor gene TP53. ${ }^{5} \mathrm{FCR}$ achieves disease control and survival prolongation, as shown in the CLL8 trial of the German CLL [chronic lymphocytic leukemia] Study Group (GCLLSG) ${ }^{3}$ and confirmed in long-term follow-up analyses. ${ }^{6-8}$ There was improvement in progression-free survival (PFS) and overall survival (OS) at a median follow-up of 5.9 years in patients treated with FCR versus FC. ${ }^{8}$ Nevertheless, patients with $\operatorname{del}(17 \mathrm{p})$ showed significantly shorter OS than all of the other genetic subgroups..$^{3,5-8}$ On the other hand, patients with $I G H V$-mutated CLL in particular seem to benefit from the addition of rituximab to chemotherapy. ${ }^{8-10}$ Common adverse events (AEs) during chemoimmunotherapy appear to be due to myelosuppression as well as immunosuppression, and the risk of secondary malignancies (myelodysplastic syndrome, acute leukemia) was 2.38 -fold higher in patients treated with FCR than in the general population. Solid tumors, exceptionally benign tumors of the skin, did not show increased incidence. ${ }^{11}$ In unfit, untreated patients without del(17p)/TP53 mutation, chlorambucil or bendamustine combined with the CD20 antibodies ofatumumab or obinutuzumab have been the standard of care. ${ }^{12,13}$ Recently, the Bruton tyrosine kinase (BTK) inhibitor ibrutinib was approved for the frontline treatment of all CLL patients, based on a randomized comparison against 
chlorambucil. ${ }^{14}$ In CLL patients with del(17p) and/or TP53 mutation in need of first-line treatment or in early relapse, novel nonchemotherapeutic agents are nowadays considered the standard of care..$^{1,9,10}$

\section{Novel agents in CLL treatment}

Ibrutinib is a first-in-class inhibitor of the BTK, covalently binding to Cys481 in BTK, resulting in prolonged target inhibition despite a short circulating half-life. ${ }^{15,16}$ It binds potently but is not fully specific, and thus inhibits other kinases like ITK. ${ }^{17}$ Ibrutinib treatment shows excellent nodal response, often along with corresponding transient lymphocytosis (referred to as "PR [partial response] with lymphocytosis"). ${ }^{18}$ To demonstrate promising results in patients treated with ibrutinib, Figure $\mathrm{S} 1$ shows OS/PFS in a Phase IB/2, openlabel, multicenter trial of patients aged at least 65 years with symptomatic CLL. For all 31 patients at 24 months, OS was 96.6\% (95\% confidence interval [CI]: 77.9\%-99.5\%) and PFS was $96.3 \%$ (95\% CI: $76.5 \%-99.5 \%){ }^{19}$ The RESONATE, ${ }^{20}$ RESONATE- $2,{ }^{14}$ and RESONATE-1 $17^{21}$ trials led to US Food and Drug Administration (FDA) and European Medicines Agency (EMA) approval. In the Phase III open-label randomized RESONATE trial, ibrutinib showed superior results concerning PFS/OS and RR to treatment with chlorambucil in previously untreated "unfit" patients aged over 65 years. ${ }^{20}$ In the untreated setting, median PFS had not been reached in the ibrutinib cohort after a median follow-up of 18.4 months, while it was 18.9 months with chlorambucil (hazard ratio: $0.16, P<0.001$; RESONATE 2 data),${ }^{14}$ and even in the relapsed or refractory $17 \mathrm{p}$-CLL setting PFS at 12 months exceeded $79 \% .{ }^{17}$ On the other hand, disease progression on ibrutinib, in particular in relapsed or refractory CLL, is difficult to treat..$^{22,23}$ As an underlying resistance mechanism, mutations were identified in Cys481, as well as activating mutations in $\mathrm{PLC} \gamma_{2}{ }^{23-25}$ Common AEs are mild diarrhea, nausea, and arthralgia. AEs led to a discontinuation rate of $10 \%-20 \%$ in clinical trials, and appeared age-dependent. ${ }^{19,25}$ In patients aged $>75$ years, atrial fibrillation and hemorrhage (probably related to inhibition of collagen-induced platelet aggregation as a side effect of BTK and Tec inhibition) appear more frequent. ${ }^{26,27}$ The second-generation BTK inhibitor acalabrutinib, irreversibly binding to BTK but not ITK or Tec, shows an overall RR (ORR) of 95\% and 10\% PR with lymphocytosis at 14.3 months, with apparently lower rates of atrial fibrillation or major hemorrhage in Phase I/II single-arm studies for pretreated CLL patients. ${ }^{28}$

Idelalisib, an inhibitor of the $\delta$-isoform of PI3 kinase (PI3K), also achieved a good ORR of $72 \%$ in Phase $\mathrm{I}^{29}$ and Phase III studies in combination with rituximab ${ }^{30}$ or ofatumumab ${ }^{31,32}$ in relapsed CLL. Immunomediated AEs (eg, hepatotoxicity, colitis, and pneumonitis) predominate, as the $\delta$-isoform of PI3K was shown to be critical for the survival and function of regulatory $\mathrm{T}$ cells. It can require drug hold and the use of corticosteroids. ${ }^{33,34}$ Younger age and mutated $I G H V$ status were significant risk factors. ${ }^{35,36}$ Recently, there have been safety issues with an increase in opportunistic and fatal infections, hepatotoxicity, and deaths ${ }^{37}$ in Phase $\mathrm{I}^{29}$ and Phase III $^{30}$ trials evaluating idelalisib in the frontline setting. In consequence, the recommendation is to use idelalisib as first-line therapy in patients harboring $\operatorname{del}(17 \mathrm{p}) /$ TP53 mutation only if other options are not available. ${ }^{38}$ As recommended by the EMA, patients under treatment with idelalisib should be given preventive medication against the lung infection Pneumocystis jirovecii pneumonia during treatment, and this should be continued for up to 6 months after treatment. ${ }^{38}$ Therefore, the use of idelalisib in the frontline setting seems to be more limited than ibrutinib. Other PI3K inhibitors are currently being tested in several trials. ${ }^{1}$

The orally available BCL2 inhibitor venetoclax, formerly called ABT199, is restoring apoptosis in tumors by directly targeting the antiapoptotic BCL2 family proteins in CLL and B-cell non-Hodgkin lymphoma (BNHL) in an inhibitory manner, acting as a $\mathrm{BH} 3$ mimetic. The BCL2-protein family includes both proapoptotic and antiapoptotic proteins, and the interplay between these groups of proteins regulates the intrinsic apoptotic pathway. The antiapoptotic proteins inhibit the activation of proapoptotic family members like Bak or Bax. ${ }^{39}$ The role of BCL2 in the survival of lymphoid malignancies is well known, and its overexpression is driven by various mechanisms. In CLL, for example, it appears to be overexpressed due to a lack of the micro-RNAs miR15a and miR16-1, which negatively regulate BCL2 expression ${ }^{40,41}$ (Figure 1). In other BNHLs (diffuse large B-cell lymphoma or follicular lymphoma), the profiling of the activity of venetoclax against a number of cell lines has identified BCL2 gains, BCL2 amplification, or the presence of the $t(14 ; 18)$ translocation as indicators of sensitivity to this agent, and can reveal potential therapeutic strategies. ${ }^{42,43}$ Here, the focus is on the profile of venetoclax and its potential in the treatment of relapsed or refractory CLL.

\section{Navitoclax and the development of venetoclax}

A first-in-class BCL2 inhibitor in human use, navitoclax, formerly called ABT263, has shown efficacy in patients with refractory CLL. It was tested in a Phase I trial without 


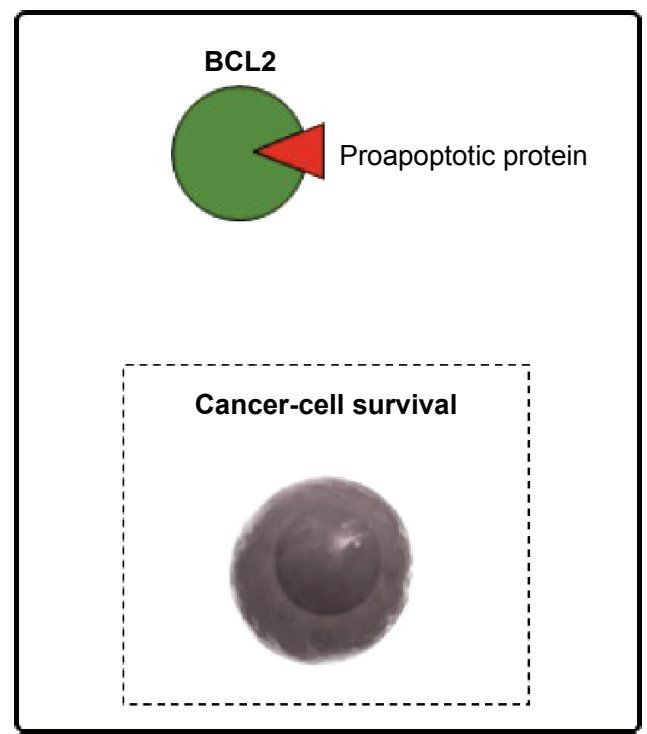

Under BCL2 overexpression cancer cells evade apoptosis by sequestering proapoptotic proteins

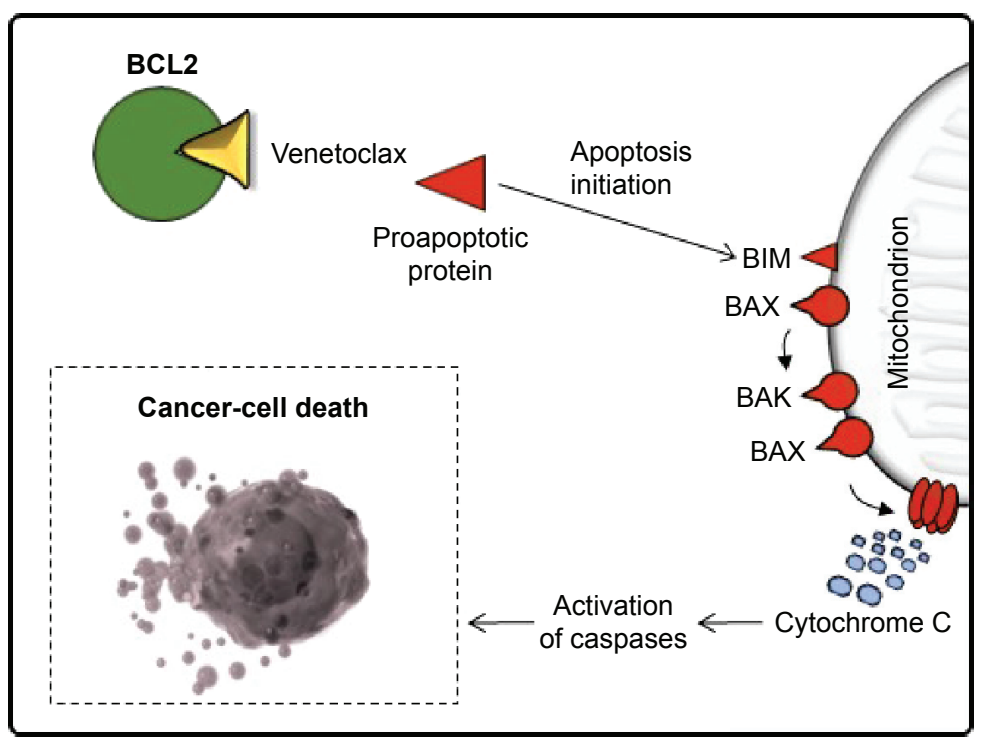

Venetoclax selectively binds to BCL2 and liberates proapoptotic proteins that initiate apoptosis

Figure I Many cancer cells are able to evade apoptosis through impairment of the mitochondrial apoptotic pathway, controlled by proapoptotic (eg, BAK, BAX, BIM) and prosurvival (eg, BCL2, BCL- $X_{L}$ ) members of the BCL2 family.

Notes: In CLL, cells show BCL2 overexpression. The BCL2 inhibitor venetoclax selectively binds to BCL2 and liberates proapoptotic proteins, inducing mitochondrial outermembrane permeabilization and leading to caspase activation. This reaction induces apoptosis.

Abbreviation: CLL, chronic lymphocytic leukemia.

special tumor-lysis management, and was designed as a dual inhibitor of both BCL2 and BCL- $\mathrm{X}_{\mathrm{L}}{ }^{44}$ However, due to the inhibition of $\mathrm{BCL}-\mathrm{X}_{\mathrm{L}}$, navitoclax induces concentrationdependent thrombocytopenia as a dose-limiting toxicity. To avoid this, a selective BCL2 inhibitor was needed, despite the high degree of similarity within the BH3-binding domains of BCL2 and BCL- $\mathrm{X}_{\mathrm{L}}{ }^{45}$

To obtain better selectivity, the structure of venetoclax (4-(4-\{[2-(2-(4-chlorophenyl)-4,4-dimethylcyclohex-1-en1-yl]methyl $\}$ piperazin-1-yl)- $N$-(\{3-nitro-4-[(tetrehydro-2Hpyrrolo[2,3-b]pyridine-5-yloxy)benzamide)), in comparison to navitoclax, lacks a thiophenyl unit in the so-called P4 hotspot. Therefore, the P4 hotspot of this new "BCL2-2 complex" provides space for a tryptophan side chain that links it to a second BCL2 protein. The nitrogen of the indole forms a hydrogen bond with Asp103 of BCL2 in the $\mathrm{BH} 3$-binding domain, which represents a major difference between venetoclax and navitoclax..$^{45}$ Due to the reengineered $\mathrm{BH} 3$-binding domain, venetoclax shows higher selectivity for BCL2, while navitoclax targets both BCL2 and BCL-X . $_{\text {. }}$ The reduced affinity to $B C L-X_{L}$ results in the sparing of platelets. ${ }^{45}$ Given that thrombocytopenia is a dose-limiting toxicity of navitoclax, venetoclax is better suited for broad clinical application in CLL.

In four mouse xenograft models, venetoclax induces apoptosis, especially in CLL cells, and furthermore shows tumor regression. It has been used as a single agent or in combination with chemotherapy without adverse reactions. ${ }^{46}$ In early clinical development, tumor-lysis syndrome (TLS) was detected as one of the primary AEs. ${ }^{46}$ The comparison of efficacy and safety profiles between navitoclax and venetoclax has revealed a potent antileukemic effect and less antiplatelet activity with venetoclax, with better compatibility in the clinical setting. ${ }^{46,47}$

\section{Phase I first-in-human trial}

A Phase I first-in-human dose-escalation clinical trial was performed for dose finding of venetoclax in patients with relapsed or refractory CLL, small LL, or BNHL requiring therapy. ${ }^{48}$ Primary objectives of this trial were the determination of safety profile and pharmacokinetic profile and evaluation of the maximum tolerated dose to develop a potential treatment schedule for a Phase II trial. Secondary end points were assessment of RRs and other markers of effectiveness. Baseline characteristics of the patients are given in Table S1. ${ }^{48}$ This trial was divided into two groups: a dose-escalation cohort, in which patients received sequentially higher doses after a minimum of three patients had completed 3 weeks of treatment without dose-limiting toxicities; and an expansion cohort, in which patients received therapy according to a predefined ramp-up plan. In the doseescalation cohort, 56 patients were enrolled in eight groups treated with escalating doses: after a test dose $(20 \mathrm{mg}$ or 
$50 \mathrm{mg}$ venetoclax) and in the absence of TLS, venetoclax was increased to the designated dose (150 mg, up to $1,200 \mathrm{mg}$ ) with stepwise weekly increases. In the expansion cohort, 60 patients were treated with weekly stepwise intrapatient dose ramp up from $20 \mathrm{mg}$ per day to $50 \mathrm{mg}, 100 \mathrm{mg}$, and $200 \mathrm{mg}$ up to $400 \mathrm{mg}$ per day (Figure S2). The majority of the patients $(89 \%)$ had poor prognostic clinical (resistance to fludarabine $60 \%$, bulky nodes $>5 \mathrm{~cm} 58 \%$ ) or genetic features (del(17p), 30\%, del(11q), 27\%, unmutated $I G H V$ $45 \%$ ). Venetoclax was active at all dose levels. It induced reduction of tumor burden in all tissue compartments, while side effects were limited. Most common AEs were nausea, diarrhea, and upper-respiratory tract infections, and were of mostly low grade and self-limiting. Hematologic AEs of Common Terminology Criteria for Adverse Events grade 3 or 4 were neutropenia (41\%), followed by thrombocytopenia $(12 \%)$ and anemia $(12 \%)$. Neutropenia was primarily observed in heavily pretreated patients. ${ }^{48}$

For TLS risk stratification, three risk categories (low, medium, and high) were defined in this trial: low risk was defined as the presence of lymph nodes with largest diameter $<5 \mathrm{~cm}$ by radiological assessment and absolute lymphocyte count $<25 \times 10^{9} / \mathrm{L}$; medium risk included only one criterion - absolute lymphocyte count $>25 \times 10^{9} / \mathrm{L}$ or a single measurable lymph node $<10 \mathrm{~cm}$ and $>5 \mathrm{~cm}$ in diameter; and the high-risk category was defined as the presence of both absolute lymphocyte count $>25 \times 10^{9} / \mathrm{L}$ and measurable lymph node with largest diameter $>5 \mathrm{~cm}$. Another high-risk criterion is the presence of any measurable $>10 \mathrm{~cm}$ in diameter by radiological assessment. ${ }^{48}$ The most important AE was TLS as a consequence of the remarkable potency to induce apoptosis. This appeared primarily in the dose-escalation cohort: in the first three of 56 patients in the dose-escalation cohort, clinical TLS was observed after a primary single dose of $100 \mathrm{mg}$ or $200 \mathrm{mg}$ venetoclax. ${ }^{47}$ The clinically relevant TLS had severe complications: one patient had acute renal failure requiring hospitalization and dialysis; a second patient had an elevation of creatinine, which resolved within 2 days; and one patient died of sudden death after stepping up to $1,200 \mathrm{mg}$. Therefore, subsequent patients in this cohort started with a lower test dose of $20 \mathrm{mg}$ or $50 \mathrm{mg}$ and underwent a ramp up in the absence of TLS to planned doses of 150-1,200 mg per day. Laboratory-only TLS occurred in seven of 56 (13\%) patients without clinical sequelae, and occurred after administration of the first dose (200 mg in two patients, $100 \mathrm{mg}$ in one patient, and $50 \mathrm{mg}$ in four patients). ${ }^{48}$

In the expansion cohort with hospitalization of highrisk patients and prophylaxis against TLS, only one patient showed evidence for laboratory TLS, but none of the patients had clinical TLS. Multiple amendments implemented additional measures that led to better safety, due to specified TLS risk management. Specified prophylaxis (laboratory TLS monitoring during ramp-up phase at 20/50 mg before start of therapy and every 2-4 hours until 24 hours) was performed prior to and during treatment, including intravenous hydration and reduction of uric acid levels (allopurinol and/or rasburicase), and patients at high risk of TLS were hospitalized for prophylaxis and observation. ${ }^{48}$

Besides TLS, further hematologic and nonhematologic AEs and serious AEs occurred in both cohorts. Neutropenia was the leading hematologic AE. Grade 4 neutropenia was observed in 33 patients (28\%), predominantly those who already had a reduced neutrophil count at enrollment. Therefore, treatment with neutrophil growth factors was allowed to make continuous treatment with venetoclax possible. However, febrile neutropenia was reported in seven of 116 patients, and 20 of 116 patients had grade 3 or 4 infections. Nevertheless, infectious complications of neutropenia appear to be less pronounced compared to treatment with chemoimmunotherapy. ${ }^{1,2,49,50}$

The ORR was 79\% in all patients, and did not vary markedly among subgroups (ORR 77\% in dose-escalation cohort, $82 \%$ in expansion cohort), with a complete response (CR) rate of $20 \%$ in all patients (CR rate $30 \%$ in dose-escalation cohort, $10 \%$ in expansion cohort). Median duration of PFS of 25 months was shown in the dose-escalation cohort, whereas in the expansion cohort the median observation time of 17 months was too short to assess PFS reliably. At month 15 , the PFS rate was estimated to be $66 \%$ (Figure $2 \mathrm{~A}$ ). ${ }^{48}$ In patients with fludarabine resistance, an ORR of $79 \%$ was achieved, with a CR rate of $16 \%$. Patients with del( $17 \mathrm{p})$ had an ORR of $71 \%$ and a CR rate of $16 \%$, with median PFS of 16 months. Venetoclax induced minimal residual disease (MRD)-negative CRs, which are generally not seen with PI3K or BTK inhibitors ${ }^{50,51}$ (Figure 2B). ${ }^{48}$ After a median follow-up of 17 months, 41 (35\%) patients showed disease progression, of which 18 patients $(16 \%)$ had Richter's transformation. Richter's transformation appears to represent a mechanism of tumor escape from suppression of BCL2 inhibition, in particular for patients with del(17p), as it was more common within this subgroup. ${ }^{48}$

With regard to the primary study end point, a maximum tolerated dose was not identified. During short-term exposure, doses as high as $800 \mathrm{mg}$ per day were not associated with higher toxicity. ORRs appeared similar between doses of $400 \mathrm{mg}$ and 1,200 mg per day. Finally, based on 
A

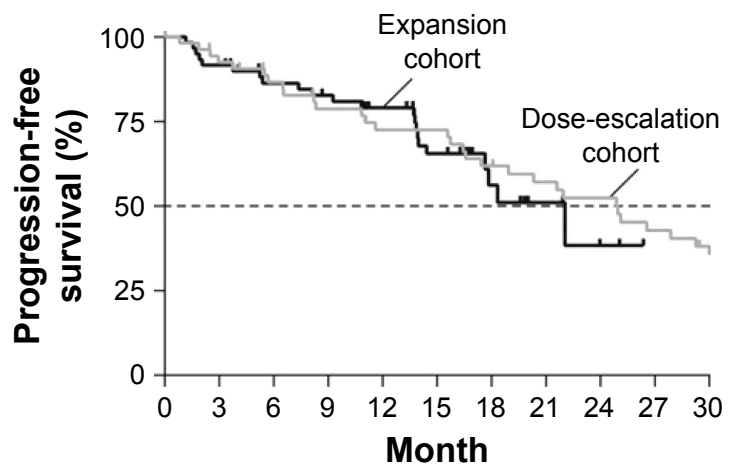

At risk, $n$

Expansion $\begin{array}{llllllllll}60 & 55 & 48 & 45 & 40 & 29 & 10 & 5 & 2\end{array}$ cohort

$\begin{array}{llllllllllll}\text { Dose-escalation } & 56 & 49 & 44 & 39 & 34 & 34 & 27 & 24 & 22 & 18 & 15\end{array}$ cohort

C

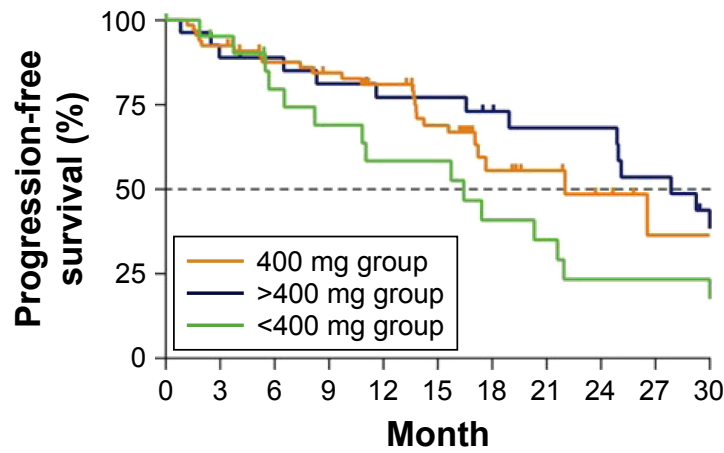

At risk, $n$

$400 \mathrm{mg}$ group $67 \begin{array}{lllllllllll}61 & 54 & 50 & 45 & 34 & 14 & 9 & 6 & 3 & 3\end{array}$ $>400 \mathrm{mg}$ group $27 \begin{array}{lllllllllll}24 & 23 & 21 & 19 & 19 & 16 & 14 & 14 & 11 & 8\end{array}$

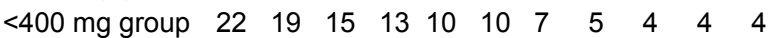

B

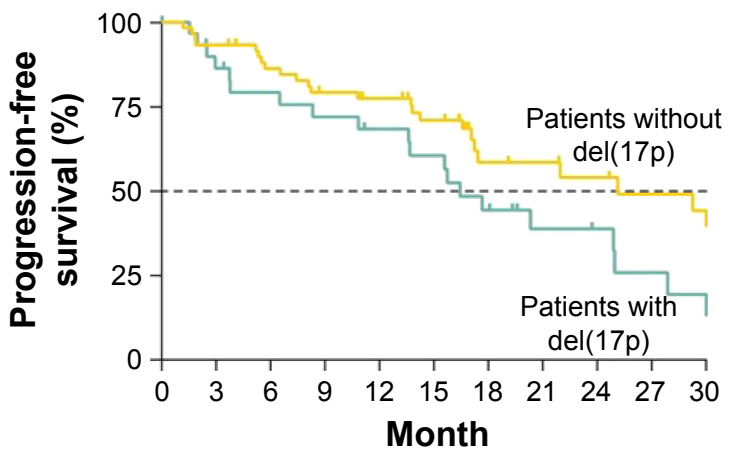

At risk, $n$

$\begin{array}{llllllllllll}\text { Patients without } & 60 & 56 & 49 & 44 & 39 & 33 & 16 & 14 & 12 & 10 & 9\end{array}$ $\operatorname{del}(17 p)$

$\begin{array}{llllllllllll}\text { Patients with } & 31 & 31 & 25 & 22 & 18 & 15 & 11 & 7 & 6 & 4 & 3\end{array}$ $\operatorname{del}(17 p)$

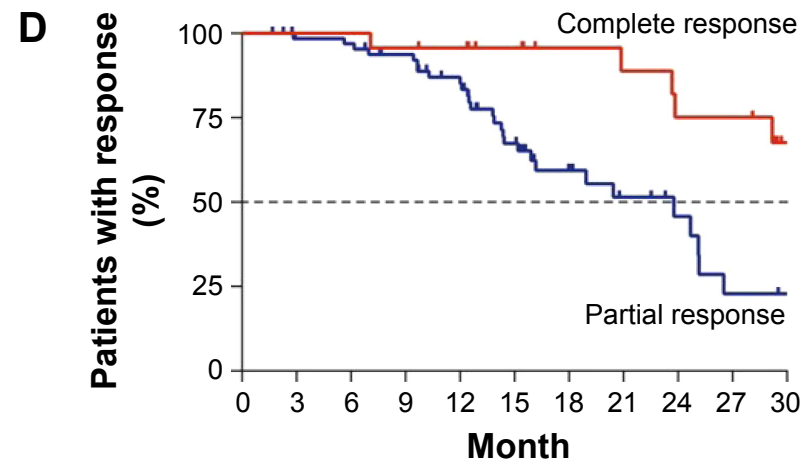

At risk, $n$

$\begin{array}{llllllllllll}\text { Complete } & 23 & 23 & 23 & 22 & 21 & 18 & 14 & 13 & 11 & 11 & 6\end{array}$ response

Partial response $6 \begin{array}{lllllllllll}69 & 63 & 62 & 56 & 48 & 32 & 18 & 12 & 8 & 4 & 3\end{array}$

Figure 2 Durability of benefit with ongoing venetoclax therapy.

Notes: (A) Progression-free survival (PFS) in the expansion and dose-escalation cohorts; (B) PFS for patients with or without del(I7p); (C) PFS and venetoclax-dose levels (400, <400, or $>400 \mathrm{mg})$; (D) duration of complete and partial response. From N Engl J Med, Roberts AW, Davids MS, Pagel JM, et al, Targeting BCL2 with venetoclax in relapsed chronic lymphocytic leukemia, 374, 31 I-322, Copyright @2016 Massachusetts Medical Society. Reprinted with permission from Massachusetts Medical Society. ${ }^{48}$

safety and efficacy data (after a minimum of 15 months of follow-up), a dose of $400 \mathrm{mg}$ per day was chosen as the target dose for further development. ${ }^{48}$ For patients receiving less than $400 \mathrm{mg}$ per day, 15-month PFS was 58\% (95\% CI: 34\%-77\%); while receiving $400 \mathrm{mg}$ per day, it reached 69\% (95\% CI: 55\%-79\%) and 77\% (95\% CI: 56\%-89\%) in patients receiving more than $400 \mathrm{mg}$ per day (Figure 2C)..$^{48}$ Among patients achieving a response, the duration of response was longer with $\mathrm{CR}$ as best response in contrast to PR (Figure 2D). ${ }^{48}$

\section{Pivotal Phase II trial}

The pivotal Phase II, open-label, multicenter study evaluated the activity and safety of venetoclax in patients with refractory or relapsed CLL harboring del(17p). A total of 107 patients were enrolled between May 2013 and June 2014. Baseline characteristics of the patients are shown in Table S2. ${ }^{52}$ Based on the results of the Phase I dose-escalation study, the patient population received a weekly dose ramp-up scheme $(20,50$, 100, 200, 400 mg, Figure S2) over 4-5 weeks. ${ }^{48}$ After dose escalation, the patients received $400 \mathrm{mg}$ venetoclax as continuous therapy until disease progression, unacceptable side effects, or other reasons for discontinuation, like subsequent allogeneic stem-cell transplantation, noncompliance, or withdrawal of consent. To manage TLS, a dedicated schedule of prophylaxis, laboratory monitoring, and hospitalization was instituted. The extent of prophylactic measures was based on tumor burden at baseline. For the initial dose-escalation steps of $20 \mathrm{mg}$ and $50 \mathrm{mg}$, all patients were hospitalized and received an oral uric acid reducer and intravenous fluids. In the following dose-escalation steps, depending on protocoldefined risk categories, patients were treated as outpatient (low and medium risk) or inpatient (high or medium risk, with low creatinine clearance), analogously to the results of 
the Phase I dose-escalation trial, according to the extent of lymphocytosis and lymphadenopathy. ${ }^{52}$ As rapid reduction of CLL cells and lymphadenopathy poses a risk of TLS during initiation of venetoclax, this risk can be reduced by lowering the initial dose and administration of intensive TLS prophylaxis. Laboratory TLS was seen in five patients during the ramp up, primarily after starting the first dose of $20 \mathrm{mg}$, and in one patient in week 3 . Only two patients needed a short interruption of 1 day each, and there were no events of clinical TLS. ${ }^{52}$

The most common AE was cytopenia. Grade 3/4 neutropenia occurred in $43(40 \%)$ patients. Venetoclax was interrupted in five (4.7\%) patients, and four (3.7\%) patients had dose reductions. Neutropenia was managed with dose interruption, GCSF application, or antibiotic prophylaxis. No patient had to discontinue venetoclax permanently due to neutropenia. Grade 3/4 anemia or thrombocytopenia occurred in $19(18 \%)$ and $16(15 \%)$ patients. Nonhematologic toxicities occurred as infection grade 3/4 in 21 (20\%) patients, in particular upper- and lower-respiratory tract infections (two patients, $2 \%$ ), pneumonia ( six patients, $6 \%$ ), and septic shock (five patients, $5 \%$ ). In ten (9\%) patients, the infection led to an interruption of venetoclax. In summary, 18 patients $(17 \%)$ died, and 11 deaths occurred in the last 30 days of treatment. Seven patients died due to disease progression, four due to AEs like septic shock or stroke. Another seven patients had disease progression after discontinuation of venetoclax (39-328 days). ${ }^{52}$

As already seen in previous trials, responses occurred early, within 1 month from the start of treatment for a majority of patients. ${ }^{48,52}$ Median time on treatment was 12.1 months, and the ORR of 85 of 107 (79.4\%) in the Phase II trial was comparable to the results of the Phase I dose-escalation trial. Median time to first response was 0.8 months, and median time to $\mathrm{CR} / \mathrm{CR}$ with incomplete recovery of blood counts (CRi) was 8.2 months (Figure 3). ${ }^{52}$ At analysis, the median was not reached for duration of OR (based on 13 events), event-free survival (34 events), time to progression (24 events), PFS (31 events), or OS (17 deaths). Estimated 12-month rates for PFS and OS were $72 \%$ and $86.7 \%$, respectively. Responses were durable, and the median duration of response was not reached (Figure 3 ). ${ }^{52}$ The majority of the patients showed reduction in absolute lymphocyte count, target lymph-node lesion diameter, and bone marrow infiltrate at a median of 0.3 months of treatment (Figure 4). ${ }^{52}$ There was a relation between the depth of the response and outcome as $\mathrm{CR} / \mathrm{CRi}$, and nodal partial remissions or MRD negativity were surrogate parameters of substantial disease reduction and associated with prolonged PFS. ${ }^{53,54}$ MRD "negativity" is a highly sensitive measure of marked reduction in disease burden, and is defined as less then one CLL cell in 10,000 leukocytes analyzed by a standard of four or more color assays with standardized-procedure flow cytometry. ${ }^{54}$ To reduce the risks of inconsistencies of local laboratories in the identification of CLL cells, it was tested at one specific regional site. MRD negativity after treatment is associated with longer disease-free intervals. MRD assessment was required in patients who achieved $\mathrm{CR}$ or CRi or who met all criteria for CR/CRi except lymphadenopathy $\leq 1.5 \mathrm{~cm}$ (but had minimally enlarged nodes of $1.5-2 \mathrm{~cm}$ ) (Figure 3 ). ${ }^{52} \mathrm{It}$ was tested by four or six color flow-cytometry assays standardized according to European Research Initiative on CLL guidelines. ${ }^{54}$ In many cases, MRD negativity was emerging beyond week 36 assessment, suggesting responses with venetoclax may further improve beyond this time point with continued dosing. In conclusion, venetoclax is able to induce MRD-negative CRs in even relapsed or refractory 17p CLL. Termination of therapy can reduce AEs, pressure to select resistance, and cost of treatment. Therefore, it is currently tested in trials whether therapy can be tailored based on MRD assessment. Although venetoclax appears very potent in inducing MRD negativity, ${ }^{51}$ there are scarce data on the durability of response after discontinuation. One trial tested the combination of rituximab and venetoclax in 49 pretreated patients, with the permission to stop venetoclax at any point desired: nine patients stopped venetoclax between months 10 and 36 from the start of therapy, and all nine remained in CR for a follow-up of 16 months. ${ }^{55}$

To examine the results of the subgroups with worse prognosis, a longer follow-up time is needed. The good response in patients with del(17p) suggests that venetoclax leads to CLL-cell death despite dysfunctional TP53. Similarly promising RRs have been shown in subgroups with other additional risk features, such as fludarabine-refractory status, bulky disease, and TP53 mutation. ${ }^{52}$ In conclusion, effective BCL2 inhibition by venetoclax appears to offer a new treatment paradigm for CLL patients harboring del(17p), in addition to ibrutinib or idelalisib, with a different efficacy and safety profile.

\section{Resistance to novel agents in CLL}

Despite the dramatic efficacy of novel agents, in some cases treatment failure occurs due to resistance and resulting disease progression. In patients treated with ibrutinib, mutations in the BTK pathway were found at the ibrutinib-binding site (BTK Cys481) with a cysteine-to-serine mutation and in 


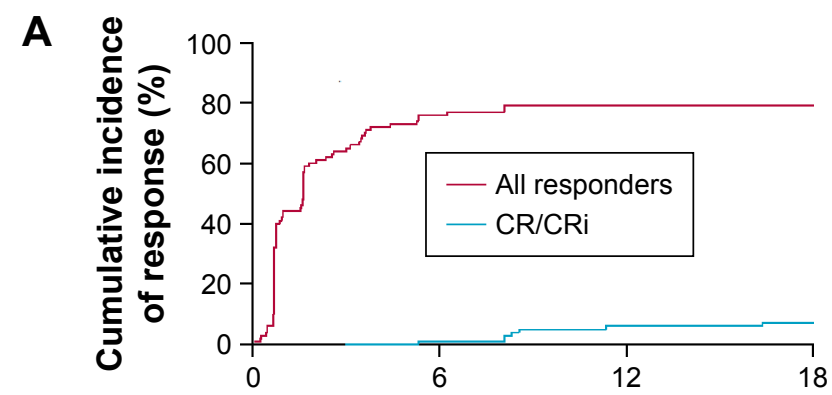

B

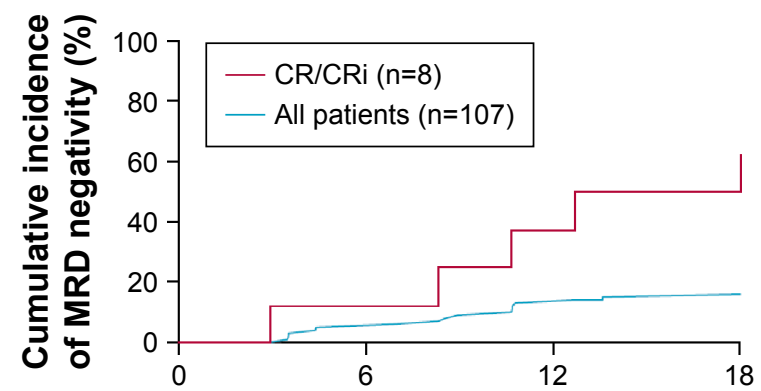

C

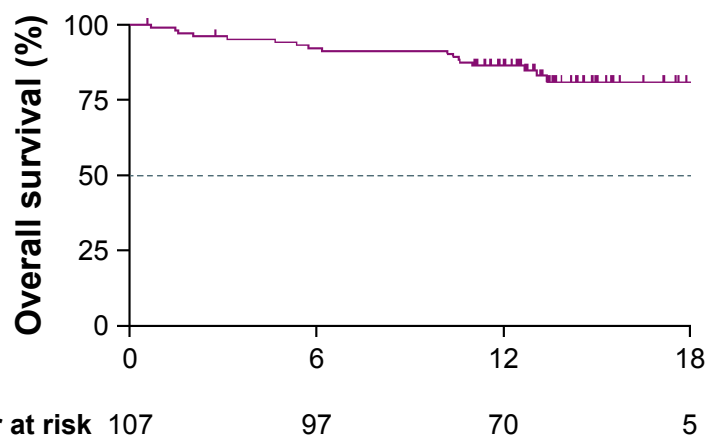

Number at risk 107

E

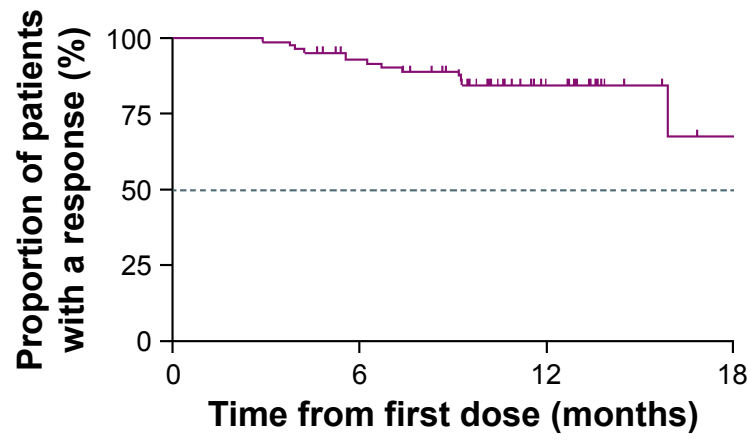

Number at risk 85
24
D

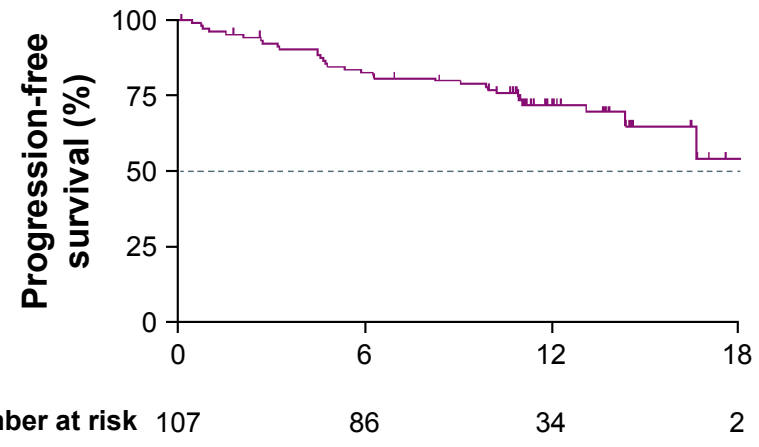

$\mathbf{F}$

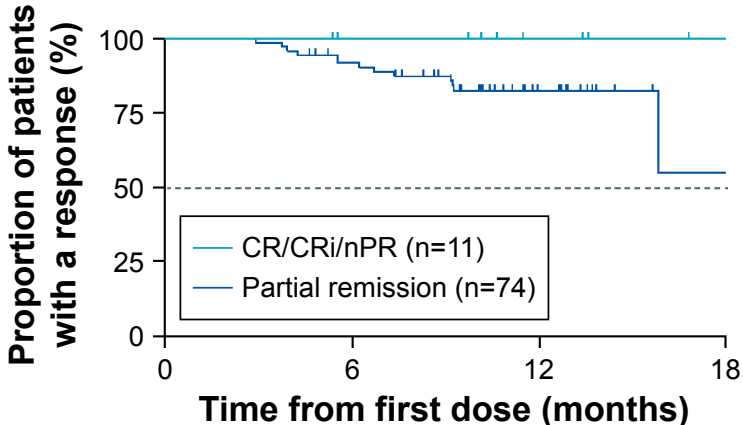

Number at risk

$\mathrm{CR} / \mathrm{CRi} / \mathrm{nPR} 11$

Partial remission 74

Time from first dose (months)

8
1
1

Figure 3 Response to venetoclax.

Notes: (A) Cumulative incidence of overall response and CR by independent review-committee assessment. (B) Cumulative incidence of minimal residual disease-negative status in peripheral blood for all patients and for patients achieving CR or CRi by independent review-committee assessment. Kaplan-Meier curves for (C) overall survival, (D) progression-free survival $(n=107)$, (E) duration of overall response for all responders by independent review-committee assessment $(n=85)$, and $(\mathbf{F})$ duration of overall response for all responders separated by response subgroups (independently assessed).

Abbreviations: $\mathrm{CR}$, complete remission; $\mathrm{CRi}, \mathrm{CR}$ with incomplete recovery of blood counts; $\mathrm{nPR}$, nodular partial response.

PLC $\gamma_{2}$ with several different presumably gain-of-function mutations. Functional characterization of these mutations demonstrated that BTK Cys418Ser reduces the binding affinity of ibrutinib for BTK, allowing only reversible BTK inhibition. ${ }^{24,56}$ The mutations identified in PLC $\gamma_{2}$ showed a potential gain of function, allowing activation in the presence of persistent BTK inhibition..$^{24,57,58}$ In contrast to ibrutinib, there are no proven resistance mechanisms in patients treated with venetoclax so far, but potential mechanisms of resistance can be supposed. One potential mechanism could be upregulation of alternative antiapoptotic BCL2 family members, such as BCL-X ${ }_{L}$, BCL-w, MCL1, and BCL2A1. ${ }^{51,59}$
ABT737, an inhibitor of BCL2 and BCL- $\mathrm{X}_{\mathrm{L}}$, induced resistance in a lymph-node model in vitro by upregulation of BCL- $\mathrm{X}_{\mathrm{L}}$ and BCL2A1. ${ }^{60}$ In a different lymph-node model, venetoclax treatment resulted in resistant CLL cells upregulating BCL- $\mathrm{X}_{\mathrm{L}}$ after CD40 and IL-4 stimulation. ${ }^{61}$ Acquired resistance after venetoclax continuous exposure in vitro was also described, and resulted in mutations in the BCL2 BH3 domain and in Bax. ${ }^{62}$

Choudhary et al developed venetoclax-resistant cell lines, exhibiting increased MCL1 stability and failing to activate Bax in response to venetoclax. The response to venetoclax was improved by the use of inhibitors of PI3K, Akt, and 

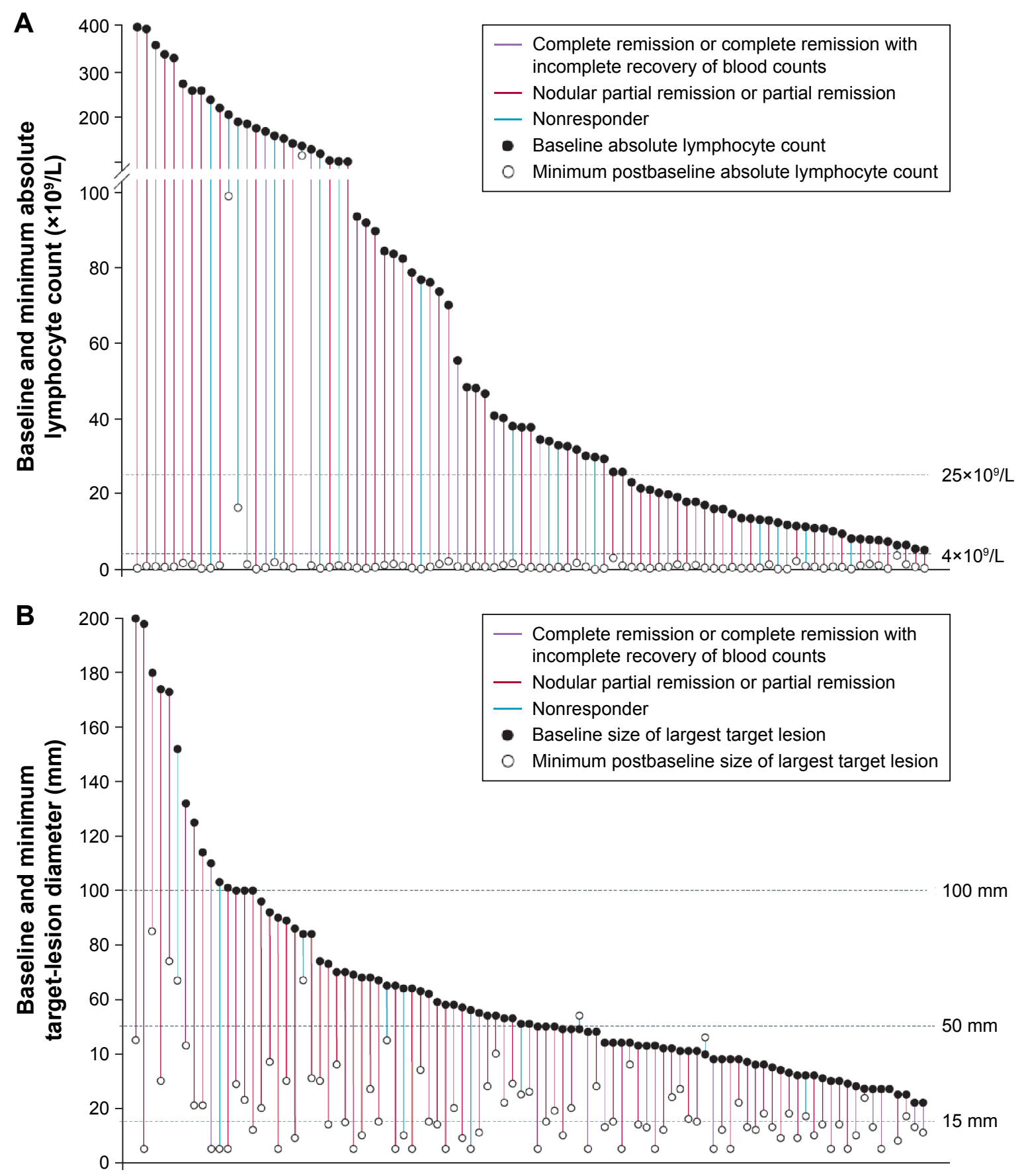

Figure 4 Venetoclax activity by compartment.

Notes: Absolute change from baseline in peripheral absolute lymphocyte count in patients with a baseline absolute lymphocyte count $>5 \times 10^{9}$ cells $/ \mathrm{L}$ ( $\left.\mathrm{n}=87\right)(\mathbf{A})$ and unidimensional nodal diameter $(\mathrm{n}=96)(\mathbf{B})$. Thresholds of $4 \times 10^{9}$ cells/L $(\mathbf{A})$ and $15 \mathrm{~mm}(\mathbf{B})$ corresponded to requirements for complete remission. Line length indicates absolute best change from baseline; each line represents one patient, with patients arranged in descending order of baseline measurement. Nodal measurements were computed tomography scan-derived unidimensional diameters of largest target lesions for patients who had at least one follow-up computed tomography scan on study. Response categories assessed by independent review committee.

mTOR pathways and idelalisib. The addition of these drugs increased the venetoclax sensitivity of CLL cells by reducing MCL1 levels and activation of Bax..$^{63}$ Oppermann et al described that venetoclax resistance, induced through upregulation of antiapoptotic BCL-X ${ }_{\mathrm{L}}, \mathrm{MCL} 1$, and BCL2A1, may be overcome by the oral, small-molecule, multitargeted receptor tyrosine-kinase inhibitor sunitinib and others to a lower extent. Sunitinib downregulates the expression of these antiapoptotic proteins, suggesting a benefit from the combination of venetoclax and sunitinib, which may be explored in early clinical trials. ${ }^{64}$ These findings need further exploration for a better understanding of potential resistance 
mechanisms, and most importantly to develop strategies to prevent or overcome them.

\section{Combination therapy in CLL}

Combinations of novel agents could achieve further synergistic activity to induce more CRs and durable MRD negativity and improve ORRs as well as OS. On the other hand, in these combination therapies, more treatment-related toxicities might appear. An overview of a selection of recruiting and ongoing clinical combination trials with venetoclax is shown in Table S3.

Navitoclax was combined with bendamustine and rituximab in lymphoma xenograft models with significant tumorgrowth control. ${ }^{65}$ Venetoclax is currently being explored in combination with antibodies, other novel compounds, and chemotherapy in different clinical trials. An open-label, multicenter Phase IB trial tested in 49 pretreated relapsed/refractory patients the combination of rituximab and venetoclax to evaluate the safety and tolerability of this treatment. The median age was 68 (range: 50-88) years, and the median number of prior regimes was two (one to five). ${ }^{55}$ In combination with rituximab, patients achieved a very good ORR of $86 \%$ (42 of 49 patients) with $41 \%$ CR/CRi (20 of 49 patients). ${ }^{66}$ Two patients showed disease progression, and one patient died before assessment (fatal TLS). Median PFS and OS were not reached. The 24-month PFS was estimated to be $84 \%$. ${ }^{66}$ $\mathrm{MRD}$ in the bone marrow was tested in 40 patients. Of those 20 patients achieving a CR/CRi, 15 of 20 (75\%) were MRDnegative tested. Overall, 26 (53\%) of 49 patients achieved MRD negativity. When achieving CR, CRi, or MRD-negative $\mathrm{PR}$, patients were permitted to stop venetoclax at any point desired. Eight patients stopped venetoclax after achieving CR/ CRi, with six also MRD-negative at the time of stopping. ${ }^{66}$ Based on these results, early stopping trials are under way.

A prospective, open-label, multicenter randomized Phase III trial CLL14 of the GCLLSG evaluated efficacy and safety of venetoclax and obinutuzumab compared with chlorambucil and obinutuzumab in the frontline setting among unfit patients. The safety run-in phase of 12 patients showed a favorable safety profile of the new combination therapy. ${ }^{67}$ Further data concerning efficacy have not been published yet, and the follow-up of the trial is ongoing.

Though ibrutinib revolutionized CLL treatment, there were still subgroups of patients without sufficient response duration, and thus venetoclax was combined with ibrutinib in vitro for further improvement. Synergistic effects were supposed, shown by the fact that the BCL2 antagonist venetoclax augmented ibrutinib's effect by targeting MCL1 and $\mathrm{BCL}-\mathrm{X}_{\mathrm{L}}$, and hence this combination targets the three antiapoptotic BCL2 family proteins supporting survival and proliferation in CLL cells. ${ }^{68}$

The MD Anderson Cancer Center is planning to activate a Phase II trial with the combination of venetoclax and ibrutinib in CLL patients (planned number of patients is about 80). Patients are eligible when refractory to and/or relapsed after at least one prior therapy. Untreated patients are eligible harboring $\operatorname{del}(17 \mathrm{p}) / T P 53$ mutation, unmutated $I G H V$, or are aged over 65 years. The primary outcome measure is best response. ${ }^{69}$

A further promising option in the treatment of CLL is the combination of monoclonal antibodies and ibrutinib. The consideration behind this is the clearance of CLL cells from peripheral blood by, for example, a CD20 antibody, while a redistribution of these cells from the lymphoid tissue to this compartment is triggered by the BTK inhibitor. This strategy is being evaluated in the CLL2-BIG trial of the GCLLSG. This trial is part of the BXX Phase II trial series evaluating the combination of a monoclonal antibody with a novel agent (BCL2 antagonist, PI3K inhibitor, BTK inhibitor). Patients with a high tumor burden receive a debulking therapy with bendamustine in advance. $^{70}$

After achievement of promising results in the combination of one novel agent with a CD20 antibody with an acceptable profile of side effects, the triple combination of a BCL2 inhibitor, a BTK inhibitor, and a CD20 antibody is being tested in further trials to improve further synergistic effects. At Ohio State University, a Phase IB/II trial is currently recruiting previously untreated or relapsed or refractory CLL patients for treatment with the triple combination of obinutuzumab, ibrutinib, and venetoclax. More than 20 patients are on treatment, the first patient cohort has already reached cycle 12, and no unexpected safety concerns have not been reported..$^{71}$

This intense triple-agent concept will also be pursued by the CLL2-GiVe Phase II study of the GCLLSG in highrisk CLL patients harboring del(17p)/TP53 mutations ${ }^{72}$ and the CLL13 study independently of genetic risk profile (in fit patients as first-line therapy vs chemoimmunotherapy, rituximab + venetoclax, and obinutuzumab + venetoclax). With the triple combination of these highly active agents with different biological mechanisms of action, the goal is to make chemotherapy obsolete in CLL management.

\section{Use of venetoclax in further hematologic malignancies and solid tumors}

As many cancer entities show significant overexpression of the antiapoptotic proteins of the BCL2 family, the use 
of venetoclax beyond CLL is a promising approach. An overview of current clinical trials in further hematologic malignancies and solid tumors is shown in Table S4 ${ }^{73,74}$ There are several ongoing studies evaluating the benefit of combination treatments especially: venetoclax in combination with bendamustine and rituximab in an ongoing Phase I trial (NCT01594229) for relapsed/refractory NHL patients (follicular, diffuse large B cell, or marginal zone lymphoma) achieved an ORR of $73 \%$ in 40 evaluable patients. In mantlecell lymphoma, venetoclax is being tested in combination with ibrutinib (Phase II study, NCT02471391), in multiple myeloma the combination of venetoclax, bortezomib, and dexamethasone is being studied (Phase I, NCT01794507), and in acute myeloid leukemia venetoclax is being used in clinical Phase I/II studies (eg, NCT02203773, NCT02287233)..$^{73}$

However, the use of venetoclax is not limited to the field of hematologic malignancies. In solid tumors, there is less experience with venetoclax, but it has been shown that BCL2 is overexpressed in $75 \%$ of breast cancers. ${ }^{74}$ In an Australian Phase I study (ISRCTN98335443), patients with estrogen receptor-positive breast cancer are being treated with venetoclax in combination with tamoxifen. A selection of current clinical trials is given in Table S2. Further data on the use of venetoclax outside CLL is awaited in the near future.

\section{Conclusion}

Novel agents have brought exceptional improvement in CLL therapy, especially in patients with del(17p) and/or TP53 mutation who traditionally had a dismal outcome after classical chemoimmunotherapy. Venetoclax was recently FDA-approved in relapsed or refractory del(17p) CLL based on impressive activity, including deep and durable remission and favorable tolerability. ${ }^{47,75}$ It adds to the growing armamentarium for the treatment of CLL with novel nonchemotherapeutic agents, such as ibrutinib and idelalisib. To improve results further, a combination of these agents appears to be an attractive approach and is currently being explored in clinical trials. First results of combination regimens can be expected in the near future, and may lead to another paradigm shift in CLL treatment approaches and ultimately cure of the disease.

\section{Disclosure}

SS has received research funding and been an advisory board member to AbbVie, Amgen, Boehringer Ingelheim, Celgene, Genentech, Genzyme, Gilead, GSK, Janssen, Mundipharma, Novartis, Pharmacyclics, and Hoffmann-La Roche. SS has non further conflicts of interest. The other authors also report no conflicts of interest in this work.

\section{References}

1. Brown JR, Hallek MJ, Pagel JM. Chemoimmunotherapy versus targeted treatment in chronic lymphocytic leukemia: when, how long, how much, and in which combination? Am Soc Clin Oncol Educ Book. 2016; 35:e387-e398.

2. Fischer K, Cramer P, Busch R, et al. Bendamustine combined with rituximab in patients with relapsed and/or refractory chronic lymphocytic leukemia: a multicenter phase II trial of the German Chronic Lymphocytic Leukemia Study Group. J Clin Oncol. 2011;29:3559-3566.

3. Hallek M, Fischer K, Fingerle-Rowson G, et al. Addition of rituximab to fludarabine and cyclophosphamide in patients with chronic lymphocytic leukaemia: a randomised, open-label, phase 3 trial. Lancet. 2010; 376:1164-1174

4. Extermann M, Overcash J, Lyman GH, Parr J, Balducci L. Comorbidity and functional status are independent in older cancer patients. $J$ Clin Oncol. 1998;16:1582-1587.

5. Döhner H, Stilgenbauer S, Benner A, et al. Genomic aberrations and survival in chronic lymphocytic leukemia. $N$ Engl J Med. 2000;343: 1910-1916.

6. Tam CS, O'Brien S, Plunkett W, et al. Long-term results of first salvage treatment in CLL patients treated initially with FCR (fludarabine, cyclophosphamide, rituximab). Blood. 2014;124:3059-3064.

7. Thomson PA, Tam CS, O'Brien SM, et al. Fludarabine, cyclophosphamide, and rituximab treatment achieves long-term disease-free survival in IGHV-mutated chronic lymphocytic leukemia. Blood. 2016;127: 303-309.

8. Fischer K, Bahlo J, Fink AM, et al. Long-term remission after FCR chemotherapy in previously untreated patients with CLL: updated results of the CLL8 trial. Blood. 2016;127:208-215.

9. Hallek M. Signaling the end of chronic lymphocytic leukemia: new frontline treatment strategies. Blood. 2013;122:3723-3734.

10. Cramer P, Hallek M, Eichhorst B. State-of-the-art treatment and novel agents in chronic lymphocytic leukemia. Oncol Res Treat. 2016; 39:25-32.

11. Benjamini $O$, Jain $P$, Trinh $L$, et al. Second cancers in patients with chronic lymphocytic leukemia who received frontline fludarabine, cyclophosphamide and rituximab therapy: distribution and clinical outcomes. Leuk Lymphoma. 2015;56:1643-1650.

12. Hillmen P, Robak T, Janssens A, et al. Chlorambucil plus ofatumumab versus chlorambucil alone in previously untreated patients with chronic lymphocytic leukaemia (COMPLEMENT 1): a randomised, multicentre, open-label phase 3 trial. Lancet. 2015;385: 1873-1883.

13. Goede V, Fischer K, Busch R, et al. Obinutuzumab plus chlorambucil in patients with CLL and coexisting conditions. N Engl J Med. 2014; 370:1101-1110.

14. Burger JA, Tedeschi A, Barr PM, et al. Ibrutinib as initial therapy for patients with chronic lymphocytic leukemia. N Engl J Med. 2015; 373:2425-2437.

15. Advani RH, Buggy JJ, Sharman JP, et al. Bruton tyrosine kinase inhibitor ibrutinib (PCI-32765) has significant activity in patients with relapsed/refractory B-cell malignancies. J Clin Oncol. 2013;31: $88-94$.

16. Honigberg LA, Smith AM, Sirisawad M, et al. The Bruton tyrosine kinase inhibitor PCI-32765 blocks B-cell activation and is efficacious in models of autoimmune disease and B-cell malignancy. Proc Natl Acad Sci U S A. 2010;107:13075-13080.

17. Dubovsky JA, Beckwith KA, Natarajan G, et al. Ibrutinib is an irreversible molecular inhibitor of ITK driving a Th1-selective pressure in T lymphocytes. Blood. 2013;122:2539-2549.

18. Byrd JC, Furman RR, Coutre SE, et al. Three-year follow-up of treatment-naïve and previously treated patients with CLL and SLL receiving single-agent ibrutinib. Blood. 2015;125:2497-2506. 
19. O'Brien S, Furman RR, Coutre SE, et al. Ibrutinib as initial therapy for elderly patients with chronic lymphocytic leukaemia or small lymphocytic lymphoma: an open-label, multicentre, phase 1B/2 trial. Lancet Oncol. 2014;15:48-58.

20. Byrd JC, Brown JR, O'Brien S, et al. Ibrutinib versus ofatumumab in previously treated chronic lymphoid leukemia. N Engl J Med. 2014; 371:213-223.

21. O'Brien S, Jones JA, Coutre S, et al. Efficacy and safety of ibrutinib in patients with relapsed or refractory chronic lymphocytic leukemia or small lymphocytic leukemia with 17 p deletion: results from the phase II RESONATE-17 trial. Blood. 2014;124:327.

22. Jain P, Keating M, Wierda W, et al. Outcomes of patients with chronic lymphocytic leukemia after discontinuing ibrutinib. Blood. 2015;125: 2062-2067.

23. Maddocks KJ, Ruppert AS, Lozanski G, et al. Etiology of ibrutinib therapy discontinuation and outcomes in patients with chronic lymphocytic leukemia. JAMA Oncol. 2015;1:80-87.

24. Woyach JA, Furman RR, Liu TM, et al. Resistance mechanisms for the Bruton's tyrosine kinase inhibitor ibrutinib. N Engl J Med. 2014; 370:2286-2294.

25. Albitar A, Ma W, De Dios I, et al. High sensitivity testing shows multiclonal mutations in patients with CLL treated with BTK inhibitor and lack of mutations in ibrutinib-naive patients. Blood. 2015;126:716.

26. Kamel S, Horton L, Ysebaert L, et al. Ibrutinib inhibits collagenmediated but not ADP-mediated platelet aggregation. Leukemia. 2014; 29:783-787.

27. Levade M, David E, Garcia C, et al. Ibrutinib treatment affects collagen and von Willebrand factor-dependent platelet functions. Blood. 2014;124:3991-3995.

28. Byrd JC, Harrington B, O'Brien S, et al. Acalabrutinib (ACP-196) in relapsed chronic lymphocytic leukemia. N Engl J Med. 2016;374:323-332.

29. Brown JR, Byrd JC, Coutre SE, et al. Idelalisib, an inhibitor of phosphatidylinositol 3-kinase p110d, for relapsed/refractory chronic lymphocytic leukemia. Blood. 2014;123:3390-3397.

30. Sharman JP, Coutre SE, Furman RR, et al. Second interim analysis of a phase 3 study of idelalisib (ZYDELIG) plus rituximab (R) for relapsed chronic lymphocytic leukemia (CLL): efficacy analysis in patient subpopulations with Del(17p) and other adverse prognostic factors. Blood. 2014 $124: 330$

31. Jones JA, Wach M, Robak T, et al. Results of a phase III randomized, controlled study evaluating the efficacy and safety of idelalisib (IDELA) in combination with ofatumumab (OFA) for previously treated chronic lymphocytic leukemia (CLL). J Clin Oncol. 2015;33 Suppl:7023.

32. Jones JA, Wach M, Robak T, et al. Updated results of a phase III randomized, controlled study of idelalisib in combination with ofatumumab for previously treated chronic lymphocytic leukemia (CLL). J Clin Oncol. 2016;34 Suppl:7515.

33. Lampson BL, Matos T, Kim HT, et al. Idelalisib given front-line for the treatment of chronic lymphocytic leukemia results in frequent and severe immune-mediated toxicities. Blood. 2015;126:497.

34. Patton DT, Garden OA, Pearce WP, et al. Cutting edge: the phosphoinositide 3-kinase p $110 \delta$ is critical for the function of CD4+CD25+Foxp3+ regulatory T cells. J Immunol. 2006;177:6598-6602.

35. Coutre S, Barrientos JC, Brown JR, et al. Safety of idelalisib in B-cell malignancies: integrated analysis of eight clinical trials. J Clin Oncol. 2015;33 Suppl:e18030.

36. Lampson BL, Kasar SN, Matos TR, et al. Idelalisib given front-line for treatment of chronic lymphocytic leukemia causes frequent immunemediated hepatotoxicity. Blood. 2016;128:195-203.

37. Barrientos JC. Idelalisib for the treatment of indolent non-Hodgkin lymphoma: a review of its clinical potential. Onco Targets Ther. 2016; 9:2945-2953.

38. European Medicines Agency. CHMP confirms recommendations for use of Zydelig. 2016. Available from: http://www.ema.europa eu/ema/index.jsp?curl=pages/medicines/human/referrals/Zydelig/ human_referral_prac_000055.jsp\&mid=WC0b01ac05805c516f. Accessed November 17, 2016.
39. Marschitz I. Analysis of Bcl-2 protein expression in chronic lymphocytic leukemia. Am J Clin Pathol. 2000;113:219-229.

40. Samuel S, Tumilasci VF, Oliere S, et al. VSV oncolysis in combination with the BCL-2 inhibitor obatoclax overcomes apoptosis resistance in chronic lymphocytic leukemia. Mol Ther. 2010;18:2094-2103.

41. Campàs C, Cosialls AM, Barragán M, et al. Bcl-2 inhibitors induce apoptosis in chronic lymphocytic leukemia cells. Exp Hematol. 2006;34: 1663-1669.

42. Tsujimoto Y, Cossman J, Jaffe E, Croce CM. Involvement of the bcl-2 gene in human follicular lymphoma. Science. 1985;228: 1440-1443.

43. Iqbal J, Nepalli VT, Wright G, et al. BCL2 expression is a prognostic marker for the activated B-cell-like type of diffuse large B-cell lymphoma. J Clin Oncol. 2006;24:961-968.

44. Wilson WH, O’Connor A, Czuczman MS, et al. Navitoclax, a targeted high-affinity inhibitor of BCL-2, in lymphoid malignancies: a phase 1 dose-escalation study of safety, pharmacokinetics, pharmacodynamics, and antitumour activity. Lancet Oncol. 2010;11:1149-1159.

45. Roberts, AW, Seymour JF, Brown JR, et al. Substantial susceptibility of chronic lymphocytic leukemia to BCL2 inhibition: results of a phase I study of navitoclax in patients with relapsed or refractory disease. J Clin Oncol. 2012;30:488-496.

46. Souers AJ, Leverson JD, Boghaert ER, et al. ABT-199, a potent and selective BCL-2 inhibitor, achieves antitumor activity while sparing platelets. Nat Med. 2013;19:202-208.

47. Leverson JD, Phillips DC, Mitten MJ, et al. Exploiting selective BCL-2 family inhibitors to dissect cell survival dependencies and define improved strategies for cancer therapy. Sci Transl Med. 2015;7:279ra40.

48. Roberts AW, Davids MS, Pagel JM, et al. Targeting BCL2 with venetoclax in relapsed chronic lymphocytic leukemia. N Engl J Med. 2016; 374:311-322.

49. Robak T, Dmoszynska A, Solal-Céligny P, et al. Rituximab plus fludarabine and cyclophosphamide prolongs progression-free survival compared with fludarabine and cyclophosphamide alone in previously treated chronic lymphocytic leukemia. J Clin Oncol. 2010;28: 1756-1765.

50. Wierda W, O'Brien S, Wen S, et al. Chemoimmunotherapy with fludarabine, cyclophosphamide, and rituximab for relapsed and refractory chronic lymphocytic leukemia. J Clin Oncol. 2005;23:4070-4078.

51. Woyach JA, Johnson AJ. Targeted therapies in CLL: mechanisms of resistance and strategies for management. Blood. 2015;126:471-477.

52. Stilgenbauer S, Eichhorst B, Schetelig J, et al. Venetoclax in relapsed or refractory chronic lymphocytic leukaemia with $17 \mathrm{p}$ deletion: a multicentre, open-label, phase 2 study. Lancet Oncol. 2016;17: 768-778.

53. Strati P, Keating MJ, O'Brien SM, et al. Eradication of bone marrow minimal residual disease may prompt early treatment discontinuation in CLL. Blood. 2014;123:3727-3732.

54. Böttcher S, Ritgen M, Fischer K, et al. Minimal residual disease quantification is an independent predictor of progression-free and overall survival in chronic lymphocytic leukemia: a multivariate analysis from the randomized GCLLSG CLL8 trial. J Clin Oncol. 2012;30:980-988.

55. Roberts AW, Ma S, Brander DM, et al. Determination of recommended phase 2 dose of ABT-199 (GDC-0199) combined with rituximab (R) in patients with relapsed/refractory $(\mathrm{R} / \mathrm{R})$ chronic lymphocytic leukemia (CLL). Blood. 2014;124:325.

56. Cheng S, Guo A, Lu P, Ma J, Coleman M, Wang YL. Functional characterization of BTK $(\mathrm{C} 481 \mathrm{~S})$ mutation that confers ibrutinib resistance: exploration of alternative k inase inhibitors. Leukemia . 2015;29: 895-900.

57. Zhou Q, Lee GS, Brady J, et al. A hypermorphic missense mutation in PLC $\gamma 2$, encoding phospholipase $\mathrm{Cy} 2$, causes a dominantly inherited autoinflammatory disease with immunodeficiency. Am J Hum Genet. 2012;91:713-720.

58. Walliser C, Hermkes E, Schade A, et al. The phospholipase C $\gamma 2$ mutants R665W and L845F identified in ibrutinib-resistant chronic lymphocytic leukemia patients are hypersensitive to the Rho GTPase Rac2. J Biol Chem. 2016;291:22136-22148. 
59. Youle RJ, Strasser A. The BCL-2 protein family: opposing activities that mediate cell death. Nat Rev Mol Cell Biol. 2008;9:47-59.

60. Vogler M, Butterworth M, Majid A, et al. Concurrent up-regulation of BCL-XL and BCL2A1 induces approximately 1000-fold resistance to ABT-737 in chronic lymphocytic leukemia. Blood. 2009; 30(113):4403-4413.

61. Thijssen R, Geest CR, de Rooij MF, et al. Possible mechanisms of resistance to the novel $\mathrm{BH} 3$-mimetic ABT-199 in in vitro lymph node models of CLL: the role of Abl and Btk. Blood. 2013;122:4188.

62. Fresquet V, Rieger M, Carolis C, Garcia-Barchino MJ, MartinezCliment JA. Acquired mutations in BCL2 family proteins conferring resistance to the BH3 mimetic ABT-199 in lymphoma. Blood. 2014;123: 4111-4119.

63. Choudhary GS, Al-Harbi S, Mazumder S, et al. MCL-1 and BCL-xLdependent resistance to the BCL-2 inhibitor ABT-199 can be overcome by preventing PI3K/AKT/mTOR activation in lymphoid malignancies. Cell Death Dis. 2015;6:e1593.

64. Oppermann S, Ylanko J, Shi Y, et al. High-content screening identifies kinase inhibitors that overcome venetoclax resistance in activated CLL cells. Blood. 2016;128:934-947.

65. Ackler S, Mitten MJ, Chen J, et al. Navitoclax (ABT-263) and bendamustine \pm rituximab induce enhanced killing of non-Hodgkin's lymphoma tumours in vivo. Br J Pharmacol. 2012;167:881-891.

66. Ma S, Brander DM, Seymour JF, et al. Deep and durable responses following venetoclax (ABT-199/GDC-0199) combined with rituximab in patients with relapsed/refractory chronic lymphocytic leukemia: results from a phase 1B study. Blood. 2015;126:830.

67. Fischer K, Fink AM, Bishop H, et al. Results of the safety run-in phase of CLL14 (BO25323): a prospective, open-label, multicenter randomized phase III trial to compare the efficacy and safety of obinutuzumab and venetoclax (GDC0199/ABT199) with obinutuzumab and chlorambucil in patients with previously untreated CLL and coexisting medical conditions. Blood. 2015;126:496.
68. Cervantes-Gomez F, Lamothe B, Woyach J, et al. Pharmacological and protein profiling suggests venetoclax (ABT-199) as optimal partner with ibrutinib in chronic lymphocytic leukemia. Clin Cancer Res. 2015;21:3705-3715.

69. MD Anderson Cancer Center. Venetoclax and ibrutinib in patients with chronic lymphocytic leukemia (CLL). Available from: https://clinicaltrials.gov/ct2/show/NCT02756897. NLM identifier: NCT02756897. Accessed November 17, 2016.

70. Tresckow JV, Cramer P, Bahlo J, et al. CLL2-BIG: a novel treatment regime of bendamustine followed by GA101 maintenance in patients with chronic lymphocytic leukemia (CLL): interim results of a phase II trial. Blood. 2015;126:4151.

71. Ohio State University Comprehensive Cancer Center. Bcl-2 inhibitor GDC-0199 in combination with obinutuzumab and ibrutinib in treating patients with relapsed, refractory, or previously untreated chronic lymphocytic leukemia. Available from: https:/clinicaltrials.gov/ct2/show/ NCT02427451. NLM identifier: NCT02427451. Accessed November $17,2016$.

72. University of Ulm. Trial of ibrutinib plus venetoclax plus obinutuzumab in patients with CLL (CLL2-GiVe). Available from: https://clinicaltrials.gov/ct2/show/NCT02758665. NLM identifier: NCT02758665. Accessed November 17, 2016.

73. Deeks ED. Venetoclax: first global approval. Drugs. 2016;76: 979-987.

74. Merino D, Lok SW, Visvader JE. Targeting BCL-2 to enhance vulnerability to therapy in estrogen receptor-positive breast cancer. Oncogene. 2016;35:1877-1887.

75. Cramer P, Langerbeins $P$, Hallek M. Combination of targeted drugs to control chronic lymphocytic leukemia: harnessing the power of new monoclonal antibodies in combination with ibrutinib. Cancer J. 2016; 22:62-66.
OncoTargets and Therapy

\section{Publish your work in this journal}

OncoTargets and Therapy is an international, peer-reviewed, open access journal focusing on the pathological basis of all cancers, potential targets for therapy and treatment protocols employed to improve the management of cancer patients. The journal also focuses on the impact of management programs and new therapeutic agents and protocols on

\section{Dovepress}

patient perspectives such as quality of life, adherence and satisfaction. The manuscript management system is completely online and includes a very quick and fair peer-review system, which is all easy to use. Visit http://www.dovepress.com/testimonials.php to read real quotes from published authors. 